\title{
PDE-4-Hemmer verhindert Exazerbationen
}

— Die häufigsten Symptome der COPD sind Atemnot, eine exzessive Produktion von Sputum und chronischer Husten.,Phänotypisch bietet die Erkrankung ein heterogenes Bild“, sagte Prof. Helmut Teschler, Essen. Somit könnten auch die typischen Symptome zwischen den einzelnen Patienten und in Abhängigkeit vom Schweregrad der Erkrankung deutlich variieren.,,Entscheidend für die Prognose ist die Exazerbationsrate", so Teschler. Solche seien nicht nur für Patienten beängstigend und belastend, sondern führten auch zu einer Verschlechterung des Allgemeinzustandes, zu einer beschleunigten Erkrankungsprogression mit zunehmender Einschränkung der Lungenfunktion und somit zu einem erhöhten Mortalitätsrisiko.

\section{Erster hochselektiver PDE-4-Inhibitor}

Bei der Pathogenese der COPD spielen Entzündunsgmechanismen, genauer gesagt die Phosphodiesterase 4, eine wichtige Rol- le. , Die Hemmung dieses Enzyms führt zu einer Abnahme der Aktivität von Entzündungszellen“, erklärte Prof. Helgo Magnussen, Großhansdorf. Selektive PDE-4Inhibitoren könnten deshalb direkt in das Entzündungsgeschehen eingreifen.

Mit Roflumilast (Daxas ${ }^{\circledR}$ ) steht jetzt ein solcher hochselektiver PDE-4-Inhibitor als orales Medikament zur Verfügung. Die Subs-tanz wurde in zwei großen randomisierten placebokontrollierten, einjährigen doppelblinden Studien bei über 3000 Patienten mit schwerer bis sehr schwerer COPD mit einer $\mathrm{FEV}_{1}<50 \%$, chronischem Husten und Auswurf untersucht.,,Roflumilast führte zu einer statistisch signifikanten Reduktion der Rate moderater bis schwerer Exazerbationen um 17\%", so Magnussen. Das exazerbationsfreie Intervall konnte von 71 auf 81 Tage verlängert werden. Die $\mathrm{FEV} 1$ verbesserte sich signifikant um $48 \mathrm{ml}$ bei einem durchschnittlichen Ausgangs- volumen von 1,0 Liter. Auch in Kombination mit Bronchodilatatoren, und zwar sowohl mit dem Betamimetikum Salmeterol als auch mit Tiotropium, zeigte Roflumilast eine additive Wirkung. So kam es bei Patienten mit gleichzeitiger Salmeteroltherapie zu einer Verbesserung der FEV1 um 49 ml, bei gleichzeitiger Gabe von Tiotropium sogar um $80 \mathrm{ml}$.

Insgesamt wurde die Therapie gut vertragen. Nur bei ca. $5 \%$ der Patienten traten relevante Nebenwirkungen auf. Dazu gehörten Diarrhö, Übelkeit und Kopfschmerzen. In allen vorliegenden Studien fand sich auch ein Gewichtsverlust, der jedoch nur das Fettgewebe und nicht die Muskulatur betrifft und mit dem Ausgangsgewicht korreliert.

sti

Pressegespräch der Fa. Nycomed: Möglicher Paradigmenwechsel in der COPD-Therapie? 03.07.2010 in Bühl

\section{Mit Lungensport Beschwerden vorbeugen und lindern}

- Um den Circulus vitiosus von Atemnot und reaktiver Schonung über zunehmende körperliche Dekonditionierung hin zu noch mehr Atemnot zu durchbrechen, ist ein dem individuellen Leistungsniveau angepasstes regelmäßiges körperliches Kraft-, Ausdauerund Koordinationstraining für COPD-Patienten aller Schweregrade ein Muss, betonte Dr.Marc Spielmanns, Leverkusen.Die zunächst nur bei Belastung spürbare Atemnot verleitet

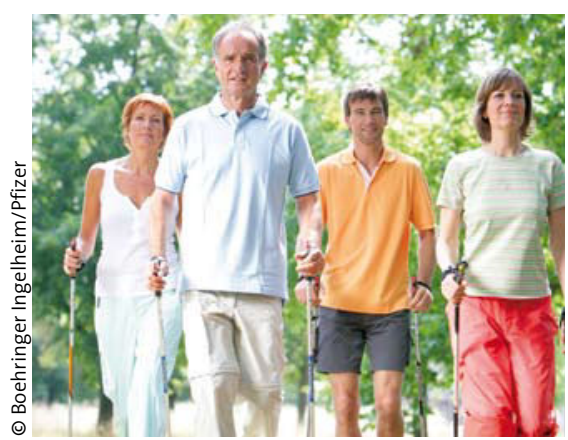

Auch Nordic Walking verbessert die Leistungsfähigkeit von COPD-Patienten.
COPD-Patienten bereits in einem frühen Stadium zu körperlicher Inaktivität. Folge ist eine zunehmende physische Dekonditionierung, die die Belastungsgrenze, ab der Atemnot auftritt, weiter senkt. Mit regelmäßigem körperlichem Training kann diese Negativspirale nicht nur verzögert, sondern in erheblichem Umfang wieder revidiert werden.

\section{Bronchodilatatoren steigern Trainingserfolg}

Mehrere Untersuchungen haben gezeigt, dass die durch Ausdauer-, Kraft-, Koordinations- und Atemtechniktraining erreichbaren Erfolge bezüglich Belastbarkeit, Dyspnoeentwicklung und Lebensqualität schon im GOLDStadium 2 durch den begleitenden Einsatz von lang wirksamen Bronchodilatatoren wie Tiotropium (Spiriva ${ }^{\circledR}$ ) maßgeblich gesteigert werden können. Fachgesellschaften empfehlen deshalb schon ab diesem moderaten Stadium zusätzlich zu regelmäßigem Lungensport lang wirksame Bronchodilatatoren als medikamentöse Basistherapie.
Um Patienten möglichst früh im Verlauf einer COPD zu dauerhaftem Lungensport und gegebenenfalls auch zur Aufgabe des Hauptrisikofaktors Rauchen zu motivieren, kann es hilfreich sein, sie und eventuell auch ihre Lebenspartner einen kleinen Selbstversuch machen zu lassen, betonte Sporttherapeut Dr. Oliver Göhl, LVA-Klinik Bad Reichenhall. Wer mit zugehaltener Nase durch einen Strohhalm mit Kaliber $5 \mathrm{~mm}$ (Obstruktionssimulation) konsequent in gehobener Atemmittellage (Emphysemsimulation) atmend erst eine halbe Minute sitze und dann noch ein paar Treppenstufen steige, bekomme eine eindrucksvolle Vorstellung, wie sich eine fortgeschrittenere COPD anfühlt, und vielleicht die Überzeugung: Es lohnt sich, etwas dafür zu tun, damit es nicht zu einer derartigen Einschränkung kommt.

ws

\section{Fachpresse-Workshop „Mehr Aktivität für COPD-Patienten" anlässlich des 42. Bad Rei- chenhaller Kolloquiums am 18.06.2010} (Veranstalter: Boehringer Ingelheim, Pfizer) 